\title{
Impact of Water on Rheological Behavior of Polyurethane Glues
}

\author{
*Laura Laiveniece, Andris Morozovs \\ Latvia University of Agriculture, Lielā street 2, Jelgava, LV-3001, Latvia
}

\begin{abstract}
Gluing of wood with great moisture content has challenge of water interaction with glue to give unwanted changes in glue properties and formed bond-line. Rheological measurements of polyurethane glues are sufficiently effective method to understand the polymer curing process and viscoelastic properties. Gel formation point is important parameter for a glue from the point of its application. When glue reaches the gel formation point, its viscosity drastically increases so that it could not properly wet the wood and it can result in poor bonds. One-component moisture curing polyurethane glues require water molecules for the polyadditional reaction. When water is involved in the curing reaction, it reacts with the isocyanate groups of glue and can lead to the formation of excessive carbon dioxide and thus can lead to glue penetration out of bond-line, additional backpressure. The dynamic viscoelastic parameters such as complex dynamic viscosity $(\eta)$, phase angle $(\delta)$, elastic storage modulus $\left(G^{\prime}\right)$, the viscous loss modulus (G”) of two kinds of polyurethane glues depending on added water amount (from $0.9 \%$ to $10.0 \%$ ) at a constant frequency and deformation (oscillation mode) were determined using rheometer. The aim of the present study was to investigate impact of added water on rheological behavior of polyurethane glues. At the end of this study it was concluded that tested glues indicated various behavior depending on chemical composition and added water content. Gel formation point is higher in case of GlueI. However, gel formation time was higher in case of GlueII and was not dependent on added water content. Gel formation time was faster for GlueII. Nevertheless, faster formed gel is stronger than longer formed gel of GlueI.
\end{abstract}

Key words: polyurethane glue, rheological behavior, curing process, green gluing.

\section{Introduction}

Polyurethane glues are used broadly in the engineered wood industry and were first approved for structural bonding for outdoor use in early 1990ies. The main advantage and at the same time the disadvantage of the one-component polyurethane glues is their curing process, which occurs at the moisture presence (Stapf, Zisi, \& Aicher, 2013). If water does not take part in reaction of glue with substrate, reaction occurs between free $-\mathrm{NCO}$ groups in prepolymer and $-\mathrm{OH}$ groups in the wood. The resulting urethane linkages lead to more complex domains which due to the potential -NCO excess, still have free reactive groups available for further reactions (Carrera et al., 2014). However, one-component moisture curing polyurethane glues require water molecules for the polyadditional reaction. Water from the wood adherends or from air moisture diffuses into the bond-line during the bonding process. Polyurethane glues cannot cure properly in bulk volumes or thick bond-lines as the outer layers of the glue that cure in an initial reaction with the ambient moisture block diffusion into the uncured core of the bulk volume (Stapf, Zisi, \& Aicher, 2013). When water is involved in the curing reaction, two more series of reactions occur. Water is known to react with the remaining isocyanate groups of glue, leading to the formation of excessive carbon dioxide and a highly reactive amine, which promotes a further reaction with isocyanate groups (Carrera et al., 2014). Excessive release of $\mathrm{CO}_{2}$ during glue curing reaction can lead to leaching out of bond-line, swelling of wood, voids in the glue, additional back pressure and it thereby can result in poor bonds. When polyurethane glue is used to make joints with green wood with moisture content above fibre saturation point, it is possible that the free water into the wood can influence gelatination and curing processes of glue.

Gelatinization (gel formation point) is an important parameter for a glue from the point of its application. Gel formation time is the maximum length of time in which the system remains sufficiently in the liquid condition (Desai et al., 2003). In gluing process wetting of the wood by glue and the penetration into it is very important. When glue reaches the gel formation point, it could not properly wet the wood and it can result in poor bonds. Gel formation point also affects open

\footnotetext{
* Corresponding Author's email: 
time in production and assembly time of constructions. Higher glue amount provides better control of the open time and the assembly time even if high amounts of water are present in green gluing case, since the larger adhesive amount implies a longer time to gel point (Stapf, Zisi, \& Aicher, 2013; Sterley et al., 2012).

Rheological measurements of glues can be used as a powerful experimental tool to monitor the polymer phase transition behavior. Rheological measurements allow to examine the properties of materials at rest state without disturbing or affecting the microstructure. Dynamic mechanical analysis is sufficiently effective method to understand the thermoset polymer curing process and to determine the viscoelastic properties of the polymer, the glass transition temperature and gel formation time (Lucio \& Fuente, 2014; Wang, Lue, \& Zhang, 2009). The formation of the polymer chain can be evaluated with the dynamic measurements assessing the elastic and viscous components of modulus respectively elastic storage and viscous loss moduli and the complex viscosity as a time depending function. (Lucio \& Fuente, 2014). The transition from a liquid to a solid structure due to gel formation point is a phenomenon of sudden solidification of polymeric liquid (Lucio \& Fuente, 2014; Wang, Lue, \& Zhang, 2009).
At the gel formation point suddenly and irreversibly viscous solution changes to a flexible gel, which is the first sign of an appearance of endless network. Macroscopic consequences of the gelatinization process is that the elastic properties of the material appear (Lucio $\&$ Fuente, 2014). Gel formation occurs at a certain critical point with a certain polymer concentration, temperature, storage time and other factors and depends on the reagent functionality, reactivity and stoichiometry. The dynamic viscoelastic parameters are important to understand and control the liquid substances in solid transition process and determine the effect of different interactions between particles of colloidal dispersion during the curing mechanism (Lucio \& Fuente, 2014; Wang, Lue, \& Zhang, 2009).

Viscosity is the fluid resistance to the flow that arises from the friction of fluid inner layers. The greater the friction between the fluid layers, the more force is needed to set them in motion. Such internal friction management mode of action is characterized by shear. Applying force $(\mathrm{F})$ to liquid layers, shear stress for a specified unit area (A) is formed; shear rate is liquid layers with height (d) displacement about the distance (u). Ratio of shear rate and shear stress is the viscosity (Figure 1) (Jaganath, 2004).
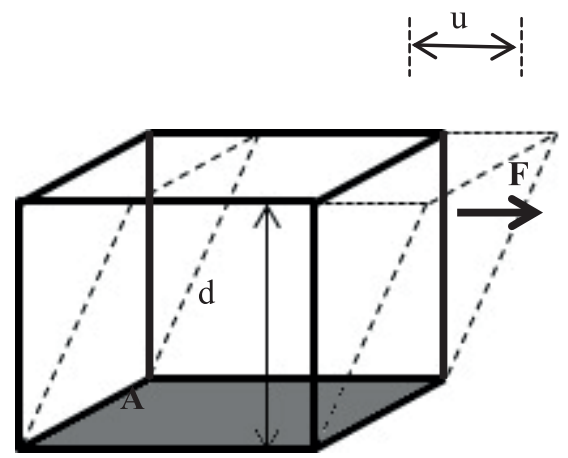

\section{Notes}

A - surface area

$\mathrm{d}$ - layer height

$\mathrm{u}$ - layer movement

$\mathrm{F}$ - applied force

Figure 1. Shear stress, shear rate and viscosity characterizing parameters (Jaganath, 2004).

The shear stress $(\tau)$ is the force $(\mathrm{F})$ and surface area $(\mathrm{A})$ ratio $\left[\mathrm{N} \mathrm{m}^{-2}=\mathrm{Pa}\right]$ (equation 1) (Jaganath, 2004):

$$
\tau=\frac{F}{A}
$$

Shear deformation $(\gamma)$ is a layer transfer $(u)$ and layer height $(d)$ ratio (equation 2) (Jaganath, 2004):

$$
\gamma=\frac{u}{d}
$$

The shear rate $(\dot{\gamma})$ is a change of shear deformation $(\mathrm{d} \gamma)$ at time interval $(\mathrm{dt})$ ratio $\left[\mathrm{s}^{-1}\right]$ (equation 3) (Jaganath, 2004):

$$
\dot{\gamma}=\frac{d \gamma}{d t}
$$

Viscosity $(\eta)$ is a shear stress $(\tau)$ and shear rate $(\dot{\gamma})$ ratio [Pa s] (equation 4) (Jaganath, 2004):

$$
\eta=\frac{\tau}{\dot{\gamma}}
$$


Rheological properties of glue (shear stress, shear deformation, viscosity, elastic storage modulus, viscous loss modulus, gel formation) may vary due to impact of various conditions (internal and external), for example, humidity or temperature (Sterley et al., 2012). In such cases, interaction between system components can be altered. With increase of interaction between system components, internal structure elements of the material will develop. These elements have the properties like a solid body and is characterized by viscoelasticity. Viscoelastic materials simultaneously combine characteristics of solid and liquid, for example, glues (Jaganath, 2004; Mezger, 2006).

Conditions that do not adversely affect the internal structure of the material are necessary to characterize properties of viscoelastic materials. When continuous shear is applied to structure (for example, using rotational viscometers) after certain interval structure would be subjected to external mechanical effects and the internal structure will be dismantled. As a result, solid particles and components of liquid phase will orient in the applied shear direction, thus overcoming yield stress and creating a flow with characteristics of fluid. Applying shear in dynamic cyclic form (for example, using oscillation mode), it is possible to deform the body to a point after which it is able to restore its initial shape and structure (Barnes, Hutton, \& Walters, 1993; Jaganath, 2004; Kim et al., 2010). The methodology of oscillation shear flow measurements allows to investigate materials not only in the liquid state, but also in rubbery and glassy state, practically including all curing process (Lucio \& Fuente, 2014).

When polyurethane glue is used to make joints with green wood with moisture content above fibre saturation point, it is possible that the free water into wood can influence gelatination and curing processes of glue. The aim of the present study was to investigate impact of added water on rheological behavior of polyurethane glues.

\section{Materials and Methods}

Two kinds of one-component moisture-cured commercial polyurethane glues were used. For the study, glues with declared water resistance class D4 and suitable for green gluing, with comparable characteristics to each other, with suitable gel formation time for experiment and are appropriate for bonding wood were selected. Fundamental characteristics of glues are summarized in Table 1.

\section{Fundamental characteristics of used glues}

\begin{tabular}{|c|c|c|}
\hline Characteristics & Glue I & Glue II \\
\hline Chemical base & Polyurethane & Polyurethane \\
\hline Curing mechanism & Moisture curing & Moisture curing \\
\hline Density, $\mathrm{g} \mathrm{cm}^{-3}$ & $\sim 1.3$ & 1.25 \\
\hline Solid residue, $\%$ & $\sim 100$ & 100 \\
\hline Viscosity, Pa s & n.d. & $6.5-9.5$ (geometry $4,10 \mathrm{rpm}$, at $+20^{\circ} \mathrm{C}$ ) \\
\hline Consumption, $\mathrm{g} \mathrm{m}^{-2}$ & 200 & $\sim 100$ \\
\hline Assembly time, min & 30 & n.d. \\
\hline Press time, $\min$ & 240 at $+20^{\circ} \mathrm{C}$ & 120 at $+20^{\circ} \mathrm{C}$ and $65 \%$ R.H \\
\hline Ultimate strength & after 24 hours at $+20^{\circ} \mathrm{C}$ & after 7 days at $+18^{\circ} \mathrm{C}$ and $60 \%$ R.H. \\
\hline Water resistance class & D4 (according to EN 2014) & D4 (according to DIN 68602/68603) \\
\hline Application areas & $\begin{array}{l}\text { For waterproof (D4) (surface) } \\
\text { bonding of practically all kinds of } \\
\text { wood. Suitable for non-bearing } \\
\text { wood constructions with various } \\
\text { connections, such as finger joints, } \\
\text { dovetails, mortise and tenon joints, } \\
\text { dowels and tongue joints. Suitable } \\
\text { for indoor and outdoor applications, } \\
\text { such as boat construction, facade } \\
\text { panelling and sandwich boards. }\end{array}$ & $\begin{array}{l}\text { Simple bonding of wood components where } \\
\text { high strength is required. The adhesive can } \\
\text { also be used for bonding or laminating many } \\
\text { other materials - wood, metals, plastic, many } \\
\text { types of semi-hard tiles, stone and cement- } \\
\text { based materials, many core materials for } \\
\text { sandwiches. }\end{array}$ \\
\hline
\end{tabular}

n.d. - data not given 
The dynamic rheology measurements of glues were determined using a constant frequency and variable deformation oscillation testing regime for viscoelastic solutions. Rheological behavior was determined by rheometer Modular Compact Rheometer MCR 302 (Anton Paar $\mathrm{GmbH}$ ) using parallel plate geometry with a diameter of $25 \mathrm{~mm}$. Test temperature using Peltier thermostatic plate was maintained at $23 \pm 0.2{ }^{\circ} \mathrm{C}$. The dynamic viscoelastic parameters such as complex dynamic viscosity $(\eta)$, phase angle $(\delta)$, elastic storage modulus $\left(G^{\prime}\right)$, the viscous loss modulus $\left(G^{\prime \prime}\right)$ at a constant frequency of $1 \mathrm{~Hz}$ and 5\% deformation (oscillation mode) were determined for each sample.

To determine rheological behavior of glues depending on water content distilled water with electrical conductivity $\leq 1.0 \mu \mathrm{S} \mathrm{cm} \mathrm{cm}^{-1}$ (A/S Spodrība), quantity from 0.9 to $10.0 \%$ was added to glues. For each glue and moisture content five parallel samples were tested. Samples were prepared in disposable $40 \mathrm{ml}$ polystyrene cups on the analytical balance. Quantity of glue was approximately 2-3 g with appropriate amount of water. The mixture was homogenized by stirring for 10-20 seconds immediately prior to testing (prior the putting on the Peltier plate). Afterwards testing was immediately started. The distance between the rotary surface and Peltier thermostatic plate or sample layer height (d) of all measurements was the same $(0.5 \mathrm{~mm})$.

\section{Results and Discussion}

During the measurements changes in viscoelastic properties such as elastic storage modulus, the viscous loss modulus and complex viscosity depending on the response time were recorded.

During dynamic mechanical analysis the examined object is dynamically deformed and simultaneously measured value of shear stress at different deformation positions depending on the measured value of the shear stress ratio versus amount of shear deformation in time (Jaganath, 2004; Mezger, 2006; Han, 2006). Sinusoidal shift between the maximal value of shear stress and the value of the shear deformation is characterized by the angle $\delta$ (Barnes, Hutton, \& Walters, 1993; Jaganath, 2004; Kim et al., 2010).

At the dynamic oscillation mode mathematical expressions of shear stress, shear rate and shear deformation are transformed, including the value of the phase shift angle. Complex shear stress is indicated by $\tau^{*}$, the complex shear deformation by $\gamma^{*}$ and the complex shear rate by $\dot{\gamma}^{*}$ (Kim et al., 2010; Mezger, 2006; Stapf, Zisi, \& Aicher, 2013). In mathematical form those parameters can be expressed with 5.-7. equations (Kim et al., 2010; Mezger, 2006):

$$
\begin{aligned}
\tau^{*} & =\tau e^{-i \delta} \\
\gamma^{*} & =\gamma e^{-i \frac{\pi}{2}} \\
\dot{\gamma}^{*} & =\dot{\gamma} e^{-i \delta}
\end{aligned}
$$

where $\delta$ - phase angle or sinusoidal shift between maximal value of shear stress and shear deformation; $i=\sqrt{-1}$. Further complex shear stress $\tau^{*}$ can be divided into $\tau^{\prime \prime}$ - the elastic shear stress component that can be expressed as $\tau \times \cos \delta$ and $\tau^{\prime}$ - viscous shear stress component that can been expressed as $\tau \times \sin \delta$. Mathematically complex shear stress can be described with equation 8 (Kim et al., 2010; Mezger, 2006):

$$
\tau^{*}=\tau^{`}-i \tau^{\prime \prime}
$$

The Hooke's law describes properties of elastic body, which indicates that the elastic body has a constant factor, which is characterized by resistance to deformation. Rheology resistance to deformation is char- acterized with a complex modulus $\mathrm{G}^{*}$, and it is given by equation 9 (Kim et al., 2010; Mezger, 2006; Stapf, Zisi, \& Aicher, 2013).

$$
G^{*}=\frac{\tau^{*}}{\gamma^{*}}=G^{\prime}+G^{\prime}
$$

Complex modulus, $\mathrm{G}^{\prime}$ and $\mathrm{G}^{\prime \prime}$ characterize material elasticity. $G^{\prime}$ is an elastic component (elastic storage modulus) of complex modulus, G" is a viscous component (viscous loss modulus) of complex modulus. In the simplified form elastic and viscous components can be described by equations 10 and 11 (Kim et al., 2010;
Mezger, 2006; Stapf, Zisi, \& Aicher, 2013). Storage modulus $G$ 'value is a measure of the deformation energy stored by the test material during the shear process. After the applied force is removed, the material energy is completely available and acting as the driving force for the reformation process which partially or complete- 
ly compensates the previously obtained deformation of the structure. Materials, storing the whole deformation energy, are showing completely reversible deformation since they are occuring finally with an unchanged shape after a load cycle. Therefore, $G^{\prime}$ represents the elastic behavior of the material. Loss modulus G" value is a measure of the deformation energy used up by the sample during the shear process. This energy is spent during the process of changing the material's structure, e.g., when the sample is flowing partially or completely. Viscoelastic flow means that there is relative motion

$$
\begin{aligned}
G^{\prime} & =\frac{\tau^{*}}{\gamma} * \cos \delta \\
G^{\prime \prime} & =\frac{\tau^{*}}{\gamma} * \sin \delta
\end{aligned}
$$

where $G^{\prime}$ is elastic storage modulus (elastic component) [Pa]; G" - viscous loss modulus (viscous component) $[\mathrm{Pa}] ; \tau^{*}$ - shear stress $[\mathrm{Pa}] ; \gamma-$ shear deformation; $\delta-$ phase angle or sinusoidal shift between maximal value of shear stress and shear deformation. Most materials, for example, glues have both components - viscous and elastic (Kim et al., 2010; Mezger, 2006; Stapf, Zisi, \& Aicher, 2013). A phase angle $\delta=0^{\circ}$ represents an ideally elastic material and $\delta=90^{\circ}$ characterizes ideally between the molecules, particles or domains, causing frictional forces between these components, and therefore, frictional heat is occuring. Energy is consumed in friction process and dissipated. A part of this energy is heating up the test material, and another part may be lost in the form of heat also to the surrounding environment. Energy losing materials are showing an irreversible deformation behavior since they occur finally with a changed shape after cycle. Therefore, G" represents the viscous behavior of the material (Mezger, 2006). viscous materials. Viscoelastic materials have a phase angle of $0^{\circ}<\delta<90^{\circ}$ (Stapf, Zisi, \& Aicher, 2013).

At constant amplitude and simultaneously constant frequency values of elastic storage and viscous loss moduli are constant. Through chemical transformation in structure, for example, in glue curing process dimers of oligomers and afterwards polymer causing the forming of structure (see Figure 2) are formed (Han, 2006).

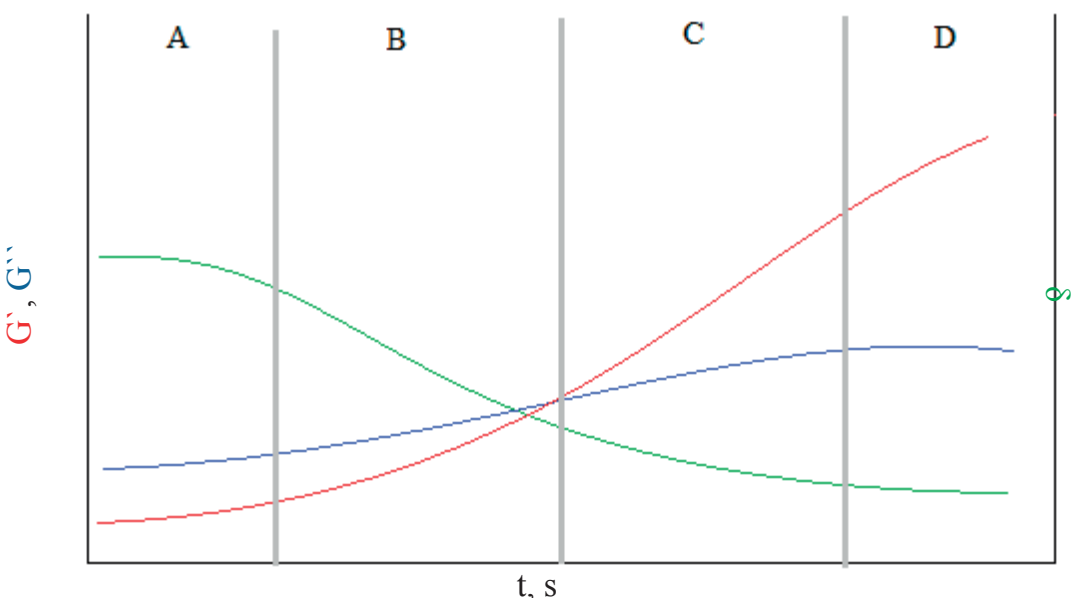

Figure 2. Shematic state transition diagram (Han, 2006).

Polymer curing process can be divided into several stages, characterized by ongoing transformations of elastic storage modulus $G^{\prime}$, viscous loss modulus G' and phase angle $\delta$ values over time. At the A stage, structure of composite is stable solution. The value of viscous loss modulus $G$ " is greater than elastic storage modulus $G^{\prime}$ and composite has characteristics of viscoelastic fluid. At the B stage, value of the viscous loss modulus $G$ " relative to elastic storage modulus $G$ ' decreases - occurs growth of oligomer chain length. Increase the proportion of agglomerates in the liquid phase and gel structure is formed. Stage of gel formation ends with the $G^{\prime}$ and $G$ " intersection (gel point). At the $\mathrm{C}$ stage, branching of polymer structure occurs, where $G$ 'value is greater than $G$ " and material has characteristics of a viscoelastic solid body. In the result of polymer crosslinking, $G^{\prime}$ value significantly increases. At the D stage, curing process of polymer is over 
(Han, 2006). Elastic storage and viscous loss moduli are temperature dependent - both moduli decrease with increase of temperature. At temperature lower than the elastic storage and viscous loss moduli cross-over point (gel formation point), the polyurethane is mainly elastic, and at temperature higher than the cross-over point, the viscous regime is dominant (García-Pacios et al., 2011).

Both moduli increase with the increase of crosslink density, but the elastic storage modulus rises more sharply than the viscous loss modulus. Such an evolution of the dynamic elastic storage and loss moduli is characteristic for networks formed by linking reaction with balanced stoichiometry. Shortly before gel formation point the material is still a liquid, so both moduli decrease to zero at low frequency. Shortly after gel formation point the elastic storage modulus tends toward a limiting value at low frequency, demonstrating the existence of permament elasticity characteristic of a solid material. The curve shapes of both $G^{\prime}(\omega)$ and $G^{\prime \prime}(\omega)$ change smoothly as the reaction proceeds, i.e.,
G' and G" come closer to each other and afterwards pass each other in a continuous fashion. At experiment for additional information, a sample at gel formation point was plunged into a mixture of methanol and toluene. The initial disklike shape of the sample dissociated almost instantaneously into numerous small clusters, revealing lack of connectivity throughout the sample and indicating a macroscopic heterogeneity in the gelation process. Shortly after gel formation point, the sample could no longer be dissolved. These experiments confirm that the crossover occurs at the instant of gelation and those rheological experiments can provide a very sensitive tool to accurately determine gel formation point (Chambon et al., 1986).

According to the Winter-Chambon criteria, the following force of law (relative change in one parameter can result in a commensurate change in another parameter) behavior exists at gel formation point (equation 12 and 13) (Chambon et al., 1986; Lucio \& Fuente, 2014; Wang, Lue, \& Zhang, 2009):

$$
\begin{aligned}
& \mathrm{G}^{\prime}(\omega)=\mathrm{G}^{\prime \prime}(\omega) \sim \omega^{\mathrm{n}} 0<\mathrm{n}<1 \\
& \mathrm{G}^{\prime \prime}(\omega) / \mathrm{G}^{\prime}(\omega)=\tan \delta=\tan (\mathrm{n} \pi / 2)
\end{aligned}
$$

where $\tan \delta$ is tangent of the loss or phase angle (see Figure 3) and n - relaxation exponent at the gel point. Phase angle $\tan \delta$ is not dependent on the frequency near to the gel formation point, and this relationship has been widely studied at the process of polymer gelatinization and improved in order to determine the gel formation point (Lucio \& Fuente, 2014; Wang, Lue, \& Zhang, 2009).

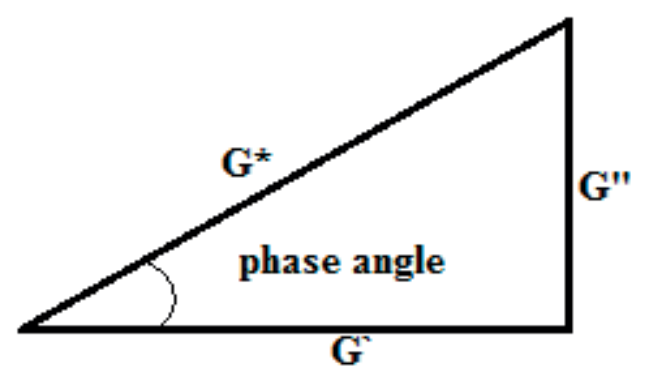

Figure 3. Phase angle schematic formation.

At the beginning of curing reaction moduli of the viscous and elastic components increase slowly and due to this fact $\tan \delta$ does not show any systematic variation in frequency. There exists critical time of aging for all samples at which $\tan \delta$ is independent on frequency. This indicates that the system has reached the gel formation point and groups of structure are macroscopically filtered according to the Winter-Chambon criteria (Lucio \& Fuente, 2014; Wang, Lue, \& Zhang, 2009). Then the elastic storage modulus, which in terms of value is less than the viscous component (viscous loss modulus) increases much faster than the other component. At the liquid phase viscous properties are dominant and more energy is dissipated than stored and G" > G' (liquid like structure). At the solid phase and when complete reaction of curing is reached, the elastic behavior dominates and more energy is stored than dissipated. In this case $\mathrm{G}^{\prime}>\mathrm{G}^{\prime}$ ' (solid like structure) (Chambon et al., 1986; Lucio \& Fuente, 2014).

During the polymer curing reactions, two transfers may occur. The first is a real chemical gelatinization, while the second is microphase separation (this transfer appears as a $\tan \delta$ maximum). Microgelatinization may occur with formation of local gel particles (Lucio \& Fuente, 2014).

During the measurements changes in viscoelas- 
tic properties such as complex dynamic viscosity (ๆ́), phase angle $(\delta)$, elastic storage modulus $\left(G^{\prime}\right)$, the viscous loss modulus ( $\left.G^{\prime \prime}\right)$ depending on the response time at a constant frequency of $1 \mathrm{~Hz}$ and $5 \%$ deformation (oscillation mode) were determined for each sample.
Gel formation point is elastic storage modulus and viscous loss modulus intersection (see Figure 4). In this study, gel formation point changes depending on added water amount were evaluated.

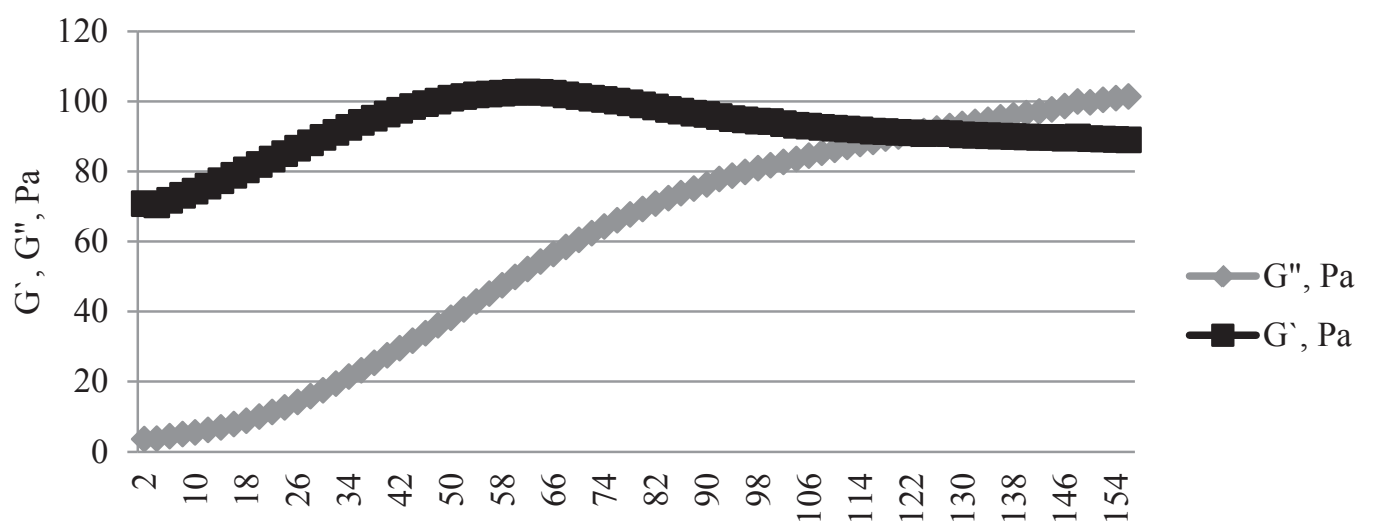

Time, $\mathrm{s}$

Figure 4. Viscous loss and elastic storage moduli values versus time.

During measurements to determine the influence of moisture content to process of glue curing the water amount from 0.9 to $10.0 \%$ was added to glue. This was intended to simulate the wide range of moisture levels: from dry to moisture contents above the wood fibre saturation point also as in other authors`(Sterley et al., 2012) research. In this study viscous loss modulus, elastic storage modulus, gel formation point, viscosity changes depending on added water amount were evaluated.

Although both glues used are one-component and based on polyurethane, their curing behavior is different.
Only its producer knows their actual chemical composition. From obtained results about gel formation point depending on added water content, we can see that gel formation point dependence from added water content can vary (see Figure 5). For GlueI obtained moderate close correlation $\left(\mathrm{R}^{2}=0.4863\right)$ between gel formation point and added water amount; for GlueII there is no relevance $\left(\mathrm{R}^{2}=0.0102\right)$ between these two parameters. Gel formation point for all added water content is higher in case of GlueI (about $10-40 \mathrm{~Pa}$ higher).

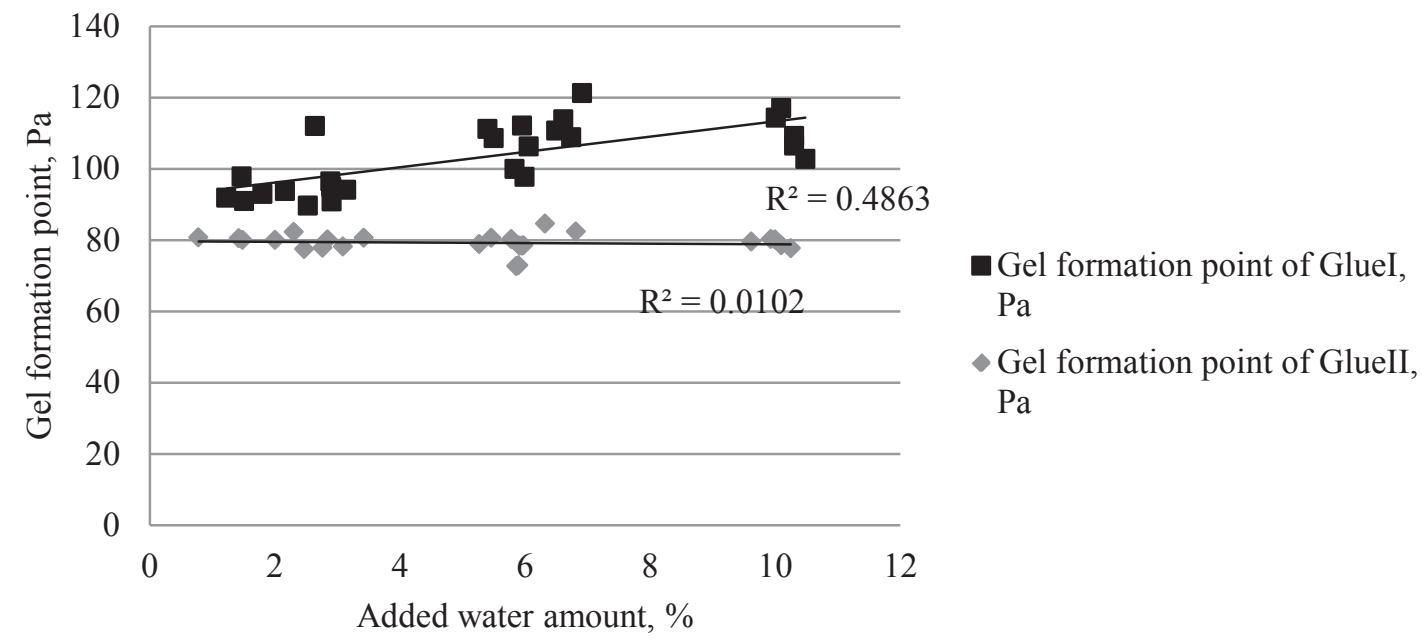

Figure 5. Gel formantion point depending on added water content. 
Test time when glues reach the gel formation point in our case does not depend on added water content (see Figure 6). Gel formation time was faster for GlueII (approximately 3 times), which indicates shorter open assembly time for joint. Unfortunately, the third tested polyurethane glue was with so fast curing mechanism that it was impossible to test it with selected test method. Therefore, only two glues were used in this study. From other authors' studies (Sterleyet al., 2012) obtained information shows that the greater the added amount of water, the faster curing speed. Time of gel formation point can affect open time in production and assembly time of constructions. In our case no effect is observed.

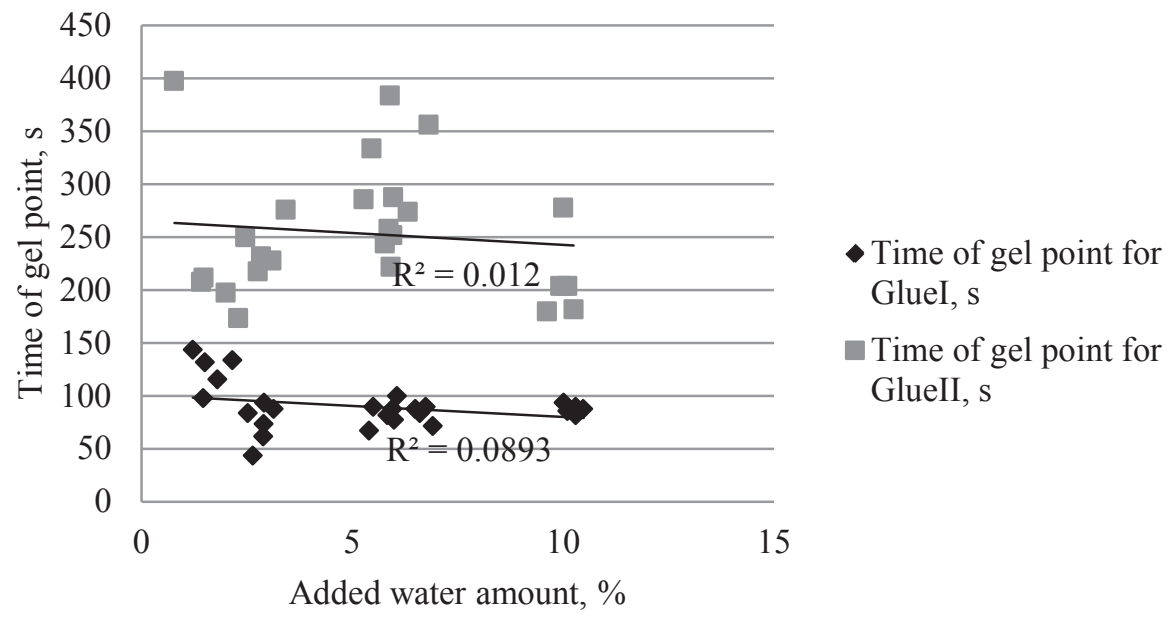

Figure 6. Gel formantion time depending on added water content.

From Sterley`s et al. studies the elastic storage modulus in the shorter period of time with water additive 2.6 and $5.6 \%$ significantly increases. However, above and below these moisture levels lower reactivity of elastic storage modulus is observed. It was concluded that water additive above the $5.6 \%$ plasticizes the polymer, resulting in a lower stiffness, but below the $2.6 \%$ water additive is too small and the elastic storage modulus increases very slowly. Nevertheless, in both cases gel formation point was reached (Sterley et al., 2012).

Basic structure of prepolymers of moisture curing one-component PU glue contains soft segments (SS) and hard segments (HS). The SS derive from the polyaddition reaction between the hydroxyl groups of more or less branched polyols and methylene diphenyl diisocyanate (MDI) isomers, forming urethane groups. The curing starts after application of glue onto the adherend and is driven by the reactivity of excess MDI isomers and moisture (from the adherend and from ambient air humidity). Carbamic acid is built as an intermediate step of this reaction, which then decomposes to a pri- mary amine and carbon dioxide gas. Consequently, the amine (or an optional chain extender) reacts with the residual isocyanate, forming urea linkages, which take effect as strong crosslinking points in the final polymer network. The areas of the polymer, which present polyurea-polyurethane groups, are referred to as HS of the cured polyurethane (see Figure 7) (Carrera et al., 2014; Kläusler et al., 2013). The mechanical characteristics of the cured polymer, like strength, stiffness or plastic workability, are derived from the characteristics of the SS, the HS and the interaction between those two by primary (covalent) bonds at the cross-linking points and additional secondary (hydrogen) bonds. The strong hydrogen bonds between the amino and carbonyl groups of urea and urethane considerably support the cohesion of the polymer matrix, whereas the hydrogen bonds between HS and polyether are much weaker. Therefore, the HS tend to form agglomerations, leading to HS and SS rich phases in the polymer. This process may be considered as a microphase separation (Kläusler et al., 2013).

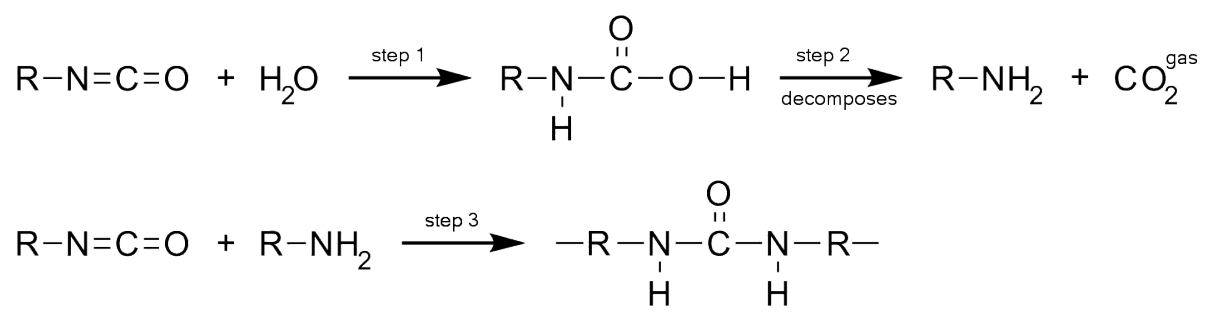

Figure 7. Urea linkage formation. 
In other authors' studies (García-Pacios et al., 2011), it was also proven that an increase in the $\mathrm{NCO} / \mathrm{OH}$ ratio of polyurethane glues produces an increase in the elastic storage modulus due to higher hard segment content and lower degree of phase separation. Similarly, the gel formation point shifts toward higher temperature and modulus as the $\mathrm{NCO} / \mathrm{OH}$ ratio increases due to the increase of the hard segments content (García-Pacios et al., 2011).

The complex viscosity of glues provides a good assessment of the viscosity during the curing reaction till gel formation point. At the beginning of curing process, complex viscosity is lower than at the gel formation point. For GlueI moderate correlation between complex viscosity at the gel formation point and added water amount - complex viscosity increases with increase of added water amount (see Figure 8) exists, while for GlueII there is practically no influence of added water amount. Complex viscosity for GlueII is more stable, even and predicted compared to GlueI. Profile of viscosity indicates that the modification of molecules due to presence of water provides significant changes of rheological behavior only for GlueI.

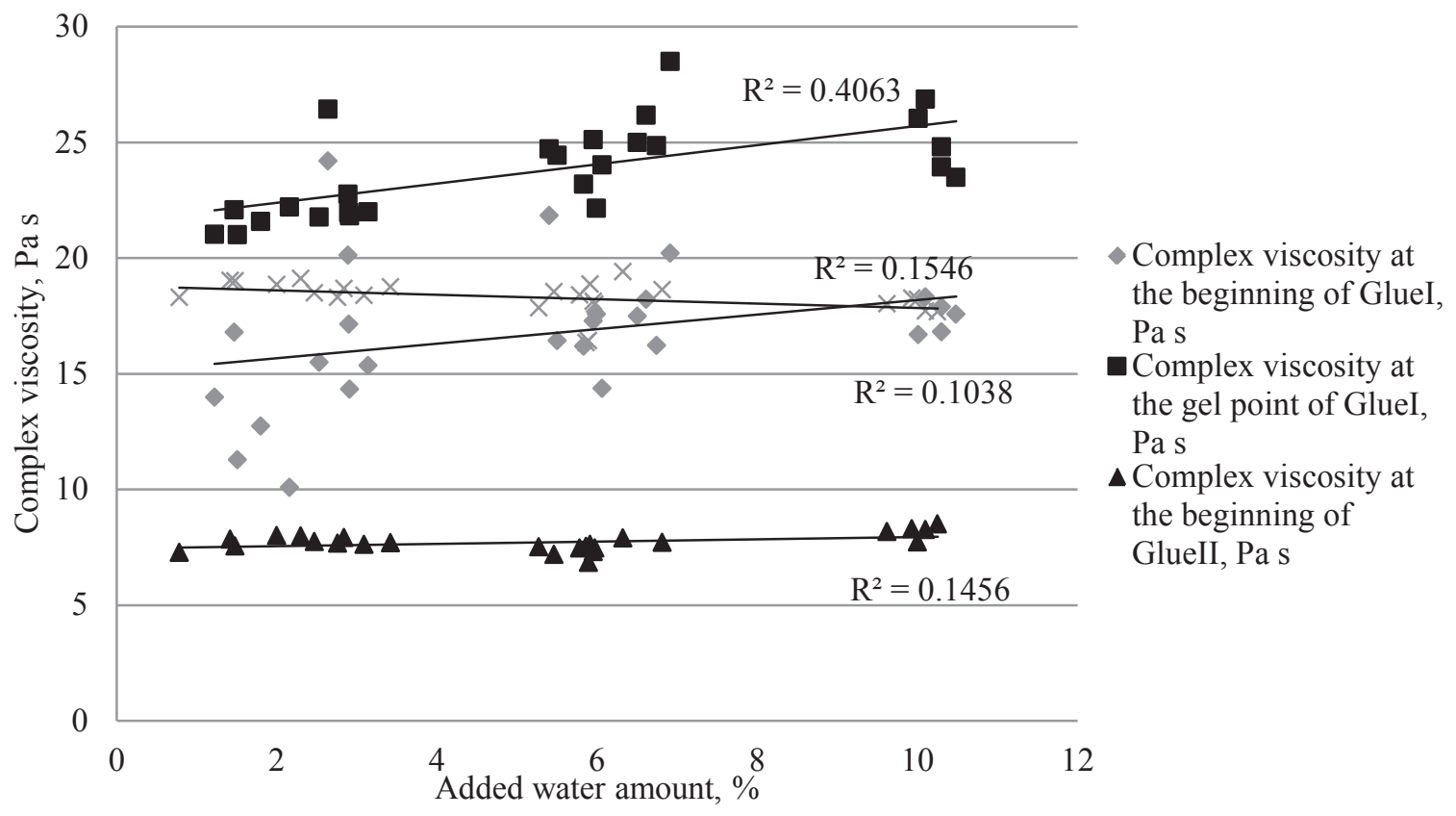

Figure 8. Complex viscosity of glues depending on added water content.

Increase of the polyurethanes functionality increases the viscosity of the system and the curing rate. Meanwhile, it causes the decrease of the time needed for the gelation time and complete curing. However, it was not found that linear correlation between the results of dynamic mechanical thermal analysis, tensile test, the gel point time and the effective functionality of the hydroxyl-terminated polybutadiene prepolymers exists (Shaghayeghi Toosi, Shahidzadeh, \& Ramezanzadeh, 2014).

Other authors (Vlad \& Oprea, 2001) conclude that viscosity linearly increases with the increase of concentration in polymeric solutions. The increase of polymeric solution concentration leads to the increase of the macromolecules interaction which determines overlapping of the chain fragments. At highenough concentra- tions, viscosity increases rapidly with the concentration increase and the shearing effects become important. The pseudoplastic behavior for specific polymer solutions is characterized by decrease of viscosity with increase of the shearing rate. As the shearing force increases, the elements start to orientate themselves along the axis, parallel to the flow direction. Interaction between elements and medium diminishes, and viscosity becomes lower than initial viscosity. At high shearing rates, viscosity reaches the minimum value and after that it remains constant (Vlad \& Oprea, 2001).

It is known that the relaxation modulus of shear $\mathrm{G}(\mathrm{t})$ are anticipated according to the force law for relaxation at gel formation point (equation 14) (Wang, Lue, \& Zhang, 2009):

$$
\mathrm{G}(\mathrm{t})=\mathrm{S}_{\mathrm{g}} \mathrm{t}^{-\mathrm{n}}
$$


where $\mathrm{S}_{\mathrm{g}}$ is the gel strength $\left[\mathrm{Pa} \mathrm{s} \mathrm{s}^{\mathrm{n}}\right]$; $\mathrm{n}$ - relaxation exponent at the gel formation point. $\mathrm{S}_{\mathrm{g}}$ is the relaxation modulus at the gel formation point, when the relaxation time ( $\mathrm{t}$ ) is 1 second. To express the gel strength as
$\mathrm{S}_{\mathrm{g}}=\mathrm{G}(\mathrm{t}) \mathrm{t}^{\mathrm{n}}$, a similar equation can be applied to moduli $G^{\prime}(\omega)$ and $G^{\prime \prime}(\omega)$ at gel formation point (equation 15) (Wang, Lue, \& Zhang, 2009):

$$
\mathrm{G}^{\prime}(\omega)=\mathrm{G}^{\prime \prime}(\omega) / \tan \delta=\mathrm{S}_{\mathrm{g}} \omega^{\mathrm{n}} \Gamma(1-\mathrm{n}) \cos (\mathrm{n} \pi / 2)
$$

where $\Gamma(1-n)$ is a function of $\Gamma$. To determine $n$ value $S_{g}$ value can be calculated from $G^{\prime}(\omega)$ or $G^{\prime \prime}(\omega)$ at gel formation point (using equation 15) (Wang, Lue, \& Zhang, 2009).

Viscoelastic nature of polymer and formation of the network structure in the system at regions before and after the gel formation point can be predicted using the dynamic mechanism analysis, based on the filtering model. In regions close to the gel formation point may reveal a significant phenomenon such characteristics as shear modulus (G', G' and complex modulus) and is expected by the force law and predictable expression of relaxation components. Shear modulus correlation with frequency is $G^{\prime} \infty G^{\prime \prime} \infty \omega^{\prime \prime}$ and $d\left(\log G^{\prime}\right) / d t \infty \omega^{-K}$ (Lucio \& Fuente, 2014).

Obtained results of gel strength depending on added water amount show very low correlation, and GlueII shows higher strength values than GlueI (see Figure 9). Added water amount does not significantly affect gel strength. Faster formed gel is with higher gel strength than longer formed gel of GlueI.

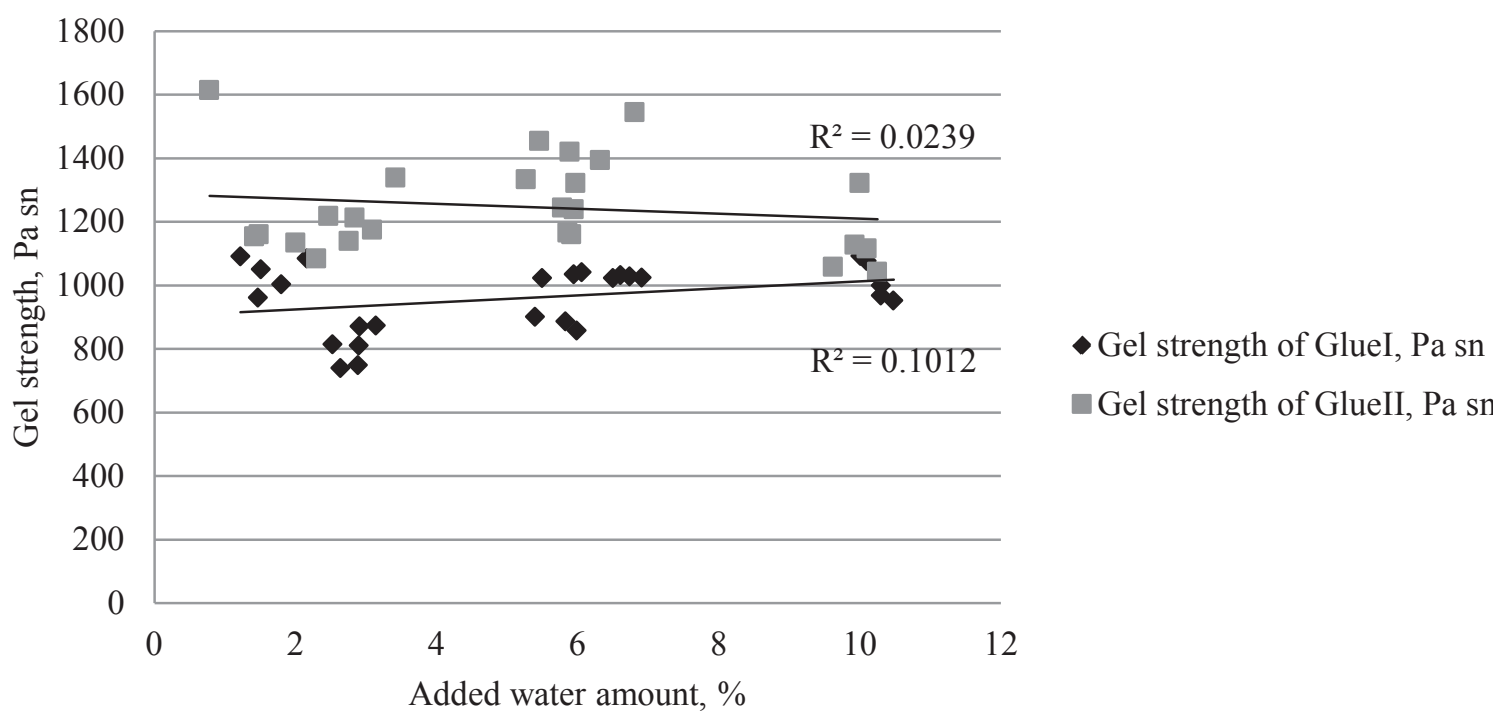

Figure 9. Gel strength depending on added water amount.

The comparatively large phase angle of the polyurethane glues during the curing process is most likely due to foaming of the adhesive because of $\mathrm{CO}_{2}$ production (Stapf, Zisi, \& Aicher, 2013). Presence of water in bond-line provides curing process of moisture-cure glues and meanwhile $\mathrm{CO}_{2}$ production, too. Phase angle increases during the cure process or phase angle at gel formation point compared to the phase angle at the beginning is shown in Figure 10. Increase of phase angle during measurement indicates that $\mathrm{CO}_{2}$ production occurs during measurement and sinusoidal shift between maximal value of shear stress and shear deformation increases. GlueII declares higher values than GlueI; however, these values are not dependent on added water amount. 


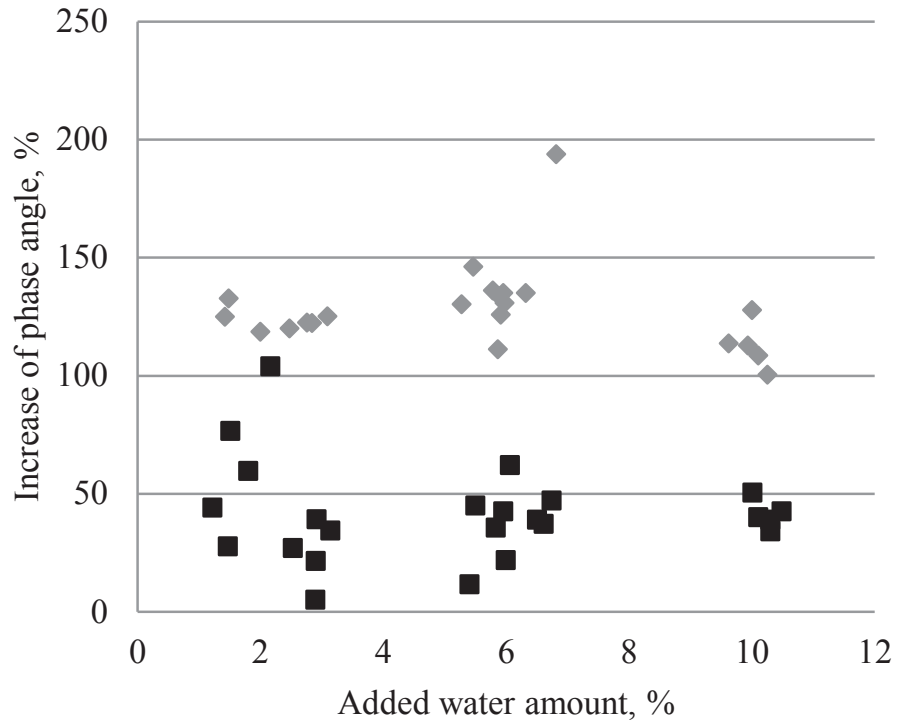

- Increase of phase angle during the cure process of GlueI, \%

- Increase of phase angle during the cure process of GlueII, \%

Figure 10. Increase of phase angle during the cure process depending on added water amount.

Obtained results of rheological behavior of glues (time to achieve the gel point, glue plasticizing with plenty of water, gel strength) can be used to develop and optimize the green wood gluing processes and better understand the mechanical properties of glue joints. In our case there is no significant influence of added water amount to polyurethane glues. When the glue reaches the gel formation point, it means that the curing mechanism of the reaction has come to a point when cross-linking of the polymer network develops. Depending on parameters of glues, wood wetting and glue penetration are affected. When viscosity is too high at gel formation point and time when gel point reached is too short, forming a weak glue joints can occur. Even if cross-linking starts to develop and forms cross-links, the presence of water from wood acts as a plasticizer and makes glue softer during curing time. It is important to avoid long open and assembly time, which may cause a risk of curing of the glue already before application of pressing pressure to joints.

\section{Conclusions}

Rheometer is a useful instrument to understand glue physical properties and curing mechanism like viscosity, viscoelastic properties, gel formation time and gel strength. Obtained results of rheological behavior of glues depending on added water content can be used to develop and optimize the green wood gluing processes and better understand the mechanical properties of glue joints. Since various polyurethane glues with different chemical composition exist, their curing behavior also varies. Tested glues also indicated various behavior depending on added water content. Gel formation point for all added water content is higher in case of GlueI (about 10 - 40 Pa higher). For GlueI obtained moderate close correlation between gel formation point and added water amount; for GlueII there is no relevance between these two parameters. However, gel formation time was higher in case of GlueII and did not depend on added water content. Test time when glues reach the gel formation point in our case is not dependent on added water content. Gel formation time was faster for GlueII (approximately 3 times), which indicates shorter open assembly time for joint. Nevertheless, faster formed gel is stronger than longer formed gel of GlueI. Presence of water in bond-line provides curing process of moisture-cure glues and meanwhile $\mathrm{CO}_{2}$ production, too. GlueII declares higher values than GlueI of phase angle increase during the cure process or phase angle at gel formation point compared to the phase angle at the beginning; however, these values are not dependent on added water amount.

\section{Acknowledgements}

The authors express their thanks to National Research Programme Forest and earth entrails resources: research and sustainable utilization - new products and technologies (ResProd) 2014 - 2017 project for financial support and to Dr.sc.ing. Ruta Galoburda for technical and scientific advice. 


\section{References}

1. Barnes, H.A., Hutton, J.F.K., \& Walters, F.R.S, (1993). An Introduction to Rheology (Third impression). Amsterdam: Elsevier B.V.

2. Carrera, V., Cuadri, A.A., García-Morales, M., \& Partal, P. (2014). Influence of the prepolymer molecular weight and free isocyanate content on the rheology of polyurethane modified bitumens. European Polymer Journal. 57, 151-159.

3. Chambon, F., Petrovic, Z.S., MacKnight, W.J., \& Winter, H.H. (1986). Rheology of model polyurethanes at the gel point. Macromolecules. 19, 21462149.

4. Desai, S.D., Patel, J.V., \&Sinha V.K. (2003). Polyurethane adhesive system from biomaterial-based polyol for bonding wood. International Journal of Adhesion \& Adhesives. 23, 393-399.

5. Jaganath, N. (2004). The Application of Reological Techniques in the Characterisation of Semisolids in the Pharmaceutical industry. Nelson Mandela Metropolitan University, Port Elizabeth.

6. García-Pacios, V., Costa, V., Colera, M., \& Martín-Martínez, J.M. (2011). Waterborne polyurethane dispersions obtained with polycarbonate of hexanediol intended for use as coating. Progress in Organic Coating. 71, 136-146.

7. Han, C.D. (2006). Rheology and Processing of Polymeric Materials. New York: Oxford University Press.

8. Kim, M.H., Min, S.H, Ferrecane, J, \& Lee, I. (2010). Initial dynamic viscoelasticity change of composites during light curing. Dental Materials. 26, 463-470.

9. Kläusler, O., Clauß, S., Lübke, L., Trachsel, J., \& Niemz P. (2013). Influence of moisture on stressstrain behaviour of adhesives used for structural bonding of wood. International Journal of Adhesion \& Adhesives. 44, 57-65.
10. Lucio, B., \& Fuente, J.L. (2014). Rheological cure characterization of an advanced functional. Thermochimica Acta. 596, 6-13.

11. Mezger, T.G. (2006). The Rheology Handbook: For users of rotational and oscillatory rheometers. ( $2^{\text {nd }}$ revised edition). Hannover: Vincentz Network.

12. Shaghayeghi Toosi, F., Shahidzadeh, M., \& Ramezanzadeh, B. (2015). An investigation of the effects of pre-polymer functionality on the curing behavior and mechanical properties of HTPBbased polyurethane. Journal of Industrial and Engineering Chemistry. 24, 166-173.

13. Stapf, G., Zisi N., \&Aicher, S. (2013). Curing behaviour of structural wood adhesives - parallel plate rheometer results. Pro Ligno.9 (4), 594-600.

14. Sterley, M., Trey, S., Lundevall, A., \& Olsson, S. (2012). Influence of cure conditions on the properties of a one-component moisture-cured polyurethane adhesive in the context of green gluing of wood. Journal of Applied Polymer Science. 126(S1), 297-304.

15. Vlad, S., \& Oprea, S. (2001). Evaluation of rheological behaviour of some thermoplastic polyurethane solutions. European Polymer Journal. 37, 2461-2464.

16. Wang, Y., Lue, A., \& Zhang, L. (2009). Rheological behavior of waterborne polyurethane/starch aqueous dispersions during cure. Polymer. 50, 5474-5481. 